\title{
Intra-aortic Balloon Pump Entrapment in a Transfemoral Sheath: Successful Management with Retrograde Transradial Wiring and Externalization
}

\author{
Giuseppe Biondi-Zoccai ${ }^{1 *}$, Massimo Mancone ${ }^{2}$, Antonino GM Marullo ${ }^{1,3}$, Mariangela Peruzzi ${ }^{1{ }^{13}}$, Elena Cavarretta ${ }^{1}$ and Giacomo Frati ${ }^{1,4}$
}

${ }^{1}$ Department of Medico-Surgical Sciences and Biotechnologies, Sapienza University of Rome, Latina, Italy

${ }^{2}$ Department of Cardiovascular, Respiratory, Nephrologic and Geriatric Sciences, Sapienza University of Rome, Rome, Italy

${ }^{3}$ Fondazione Eleonora Lorillard Spencer Cenci Foundation, Rome, Italy

${ }^{4}$ Department of AngioCardioNeurology, IRCCS Neuromed, Pozzilli, Italy

\begin{abstract}
Mechanical circulatory support by means of Intra-aortic Balloon Pump (IABP) is an established therapeutic means in high-risk patients undergoing coronary revascularization (e.g. due to severe left ventricular systolic dysfunction) or those with cardiogenic shock refractory to medical therapy. Despite its remarkable safety profile, complications during IABP deployment still occur, and may be life-threatening, especially because of the underlying risk of those receiving such device. Indeed, the IABP balloon may occasionally become entrapped in the transfemoral sheath during delivery. We hereby report a case in which IABP entrapment in a transfemoral sheath during initial delivery was successfully managed by means of retrograde wiring with an exchange-length 0.018 " guide wire delivered through a transradial sheath. This clinical vignette provides evidence that a simple technical trick (i.e. retrograde tracking of $300 \mathrm{~cm}$ guide wire between the IABP shaft and the internal wall of the delivery sheath followed by guide wire externalization) may prove effective and safe in solving this potentially dangerous complication.
\end{abstract}

Keywords: Complication; Entrapment; Femoral artery; Intra-aortic balloon pump; Radial artery

\section{Introduction}

The management of patients with or at risk of cardiogenic shock remains challenging, despite several developments in recent years including Extra-corporeal Membrane Oxygenation (ECMO) and ventricular assist devices. Indeed, intra-aortic balloon pump (IABP) remains widely employed in such subjects given its ease of use and established risk-safety profile [1]. Nonetheless, complications with IABP still occur, even if less commonly than in the past [2]. When they happen, they represent a therapeutic challenge because of the underlying patient instability and large sheath required for IABP insertion. We hereby report a case of IABP entrapment during initial deployment, which was successfully managed by means of transradial retrograde wiring and externalization.

\section{Case}

A 74-year-old man with a history of diabetes mellitus, hypertension and prior anterior myocardial infarction was admitted for NonST-Elevation Myocardial Infarction (NSTEMI). Transthoracic echocardiography showed a left ventricular ejection fraction of $25 \%$, with anterior dyskinesia and infero-lateral hypokinesia, whereas coronary angiography disclosed a chronic total occlusion of the Left Anterior Descending (LAD), a significant stenosis in the second segment of the Left Circumflex (LCX), and a subocclusive stenosis in the first segment of a dominant Right Coronary Artery (RCA). After discussion of the case with the attending cardiothoracic surgeon, and in light of the prior transmural myocardial infarction without residual myocardial viability, Percutaneous Coronary Intervention (PCI) was planned on the RCA and LCX.

The left radial artery was chosen as access site for PCI, and, given the high-risk patient features, prophylactic insertion of IABP (40 cc ACAT, Arrow International, Reading, PA, USA), was attempted through the right femoral artery with its dedicated 8 French sheath and
0.025 " guide wire. No particular resistance was found when deploying the guide wire, which indeed could thus be delivered uneventfully up to the aortic arch. Conversely, progressively increasing resistance was found when advancing the shaft of the IABP, such that after advancing it for $10 \mathrm{~cm}$, marked resistance was felt, and no further advancement of the IABP catheter was possible. Furthermore, the 0.025 " guide wire also proved entrapped and could not be advanced nor retrieved despite several forceful attempts, thus making simple guide wire exchange impossible. As the guide wire had already been delivered quite distally in the aortic arch, its J tip was free of any attrition. Even attempts to try to straighten it were not associated with any meaningful impact on balloon or guide wire entrapment. Most likely, the cause of the entrapment was kinking of the IABP balloon and hypotube, followed by kinking of the guide wire itself.

The fact that both balloon and guide wire were entrapped and could not be either advanced or retrieved made us fear that the intraluminal seating of the guide wire could be lost if the balloon was retrieved. Accordingly, in order to retrieve the IABP without losing transfemoral access in such a high-risk patient, we decided to exploit

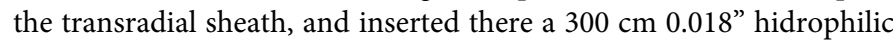
guide wire (V18 Control Wire, Boston Scientific, Natick, MA, USA). After angiography of the right external iliac and common femoral

*Corresponding author: Dr. Giuseppe Biondi Zoccai, Department of MedicoSurgical Sciences and Biotechnologies, Sapienza University of Rome, Corso della Repubblica 79, 04100 Latina, Italy, E-mail: gbiondizoccai@gmail.com

Received January 27, 2013; Accepted March 12, 2013; Published March 16, 2013

Citation: Zoccai GB, Mancone M, Marullo AGM, Peruzzi M, Cavarretta E, et al. (2013) Intra-aortic Balloon Pump Entrapment in a Transfemoral Sheath Successful Management with Retrograde Transradial Wiring and Externalization. J Cardiovasc Dis Diagn 1: 103. doi:10.4172/2329-9517.1000103

Copyright: (c) 2013 Zoccai GB, et al. This is an open-access article distributed under the terms of the Creative Commons Attribution License, which permits unrestricted use, distribution, and reproduction in any medium, provided the original author and source are credited. 
Citation: Zoccai GB, Mancone M, Marullo AGM, Peruzzi M, Cavarretta E, et al. (2013) Intra-aortic Balloon Pump Entrapment in a Transfemoral Sheath: Successful Management with Retrograde Transradial Wiring and Externalization. J Cardiovasc Dis Diagn 1: 103. doi:10.4172/23299517.1000103

arteries with a 6 French pigtail catheter (Cordis, Miami, FL, USA) enabling road-mapping (Figure 1 ; Movie 1 ), the 0.018 " guide wire was retrogradely delivered from the left radial artery, to the left brachial artery, the left axillary artery, the left subclavian artery, the descending thoracic aorta, the abdominal aorta, the right common iliac artery, the right external iliac artery, and finally within the IABP sheath (precisely between the internal wall of the sheath and the IABP balloon). Then, it was retrogradely externalized at the level of the right femoral artery (Figure 2; Movie 2), according to what is often done during endovascular procedures [3]. Afterwards, the sheath and the IABP were simultaneously retrieved and discarded, while vascular access was secured with a 5 French sheath (Cordis) delivered on the 0.018 " guide wire, enabling exchange for a new set of IABP, sheath, and 0.025 " guide wire. After control angiography showing no significant angiographic complication (Figure 3; Movie 3), a new IABP was thus successfully deployed and activated, and PCI could be carried out uneventfully by means of transradial access. The post-procedural course was satisfactory, without any additional major or minor complications. The patient could be discharged to a rehabilitation facility 3 days after PCI, with a pre-discharge duplex ultrasound of the right external iliac and common femoral arteries disclosing no significant lesions.

\section{Discussion}

Despite some recent inconclusive results, IABP is widely in patients

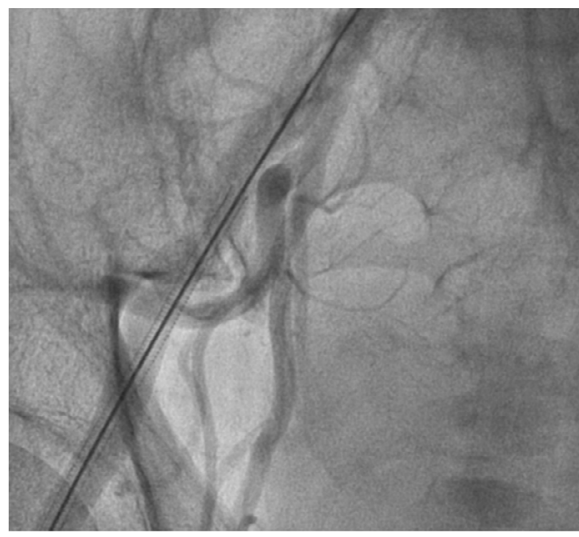

Figure 1: Angiography of the right external iliac and common femoral arteries showing the intra-aortic balloon pump stuck in the delivery sheath.

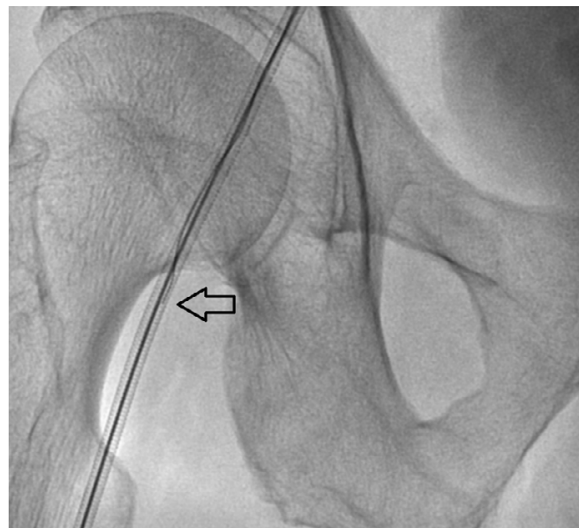

Figure 2: Fluoroscopy showing the 0.018" guidewire (arrow) delivered transradially between the internal wall of the sheath and the intra-aortic balloon pump, toward the right femoral arteriotomy.

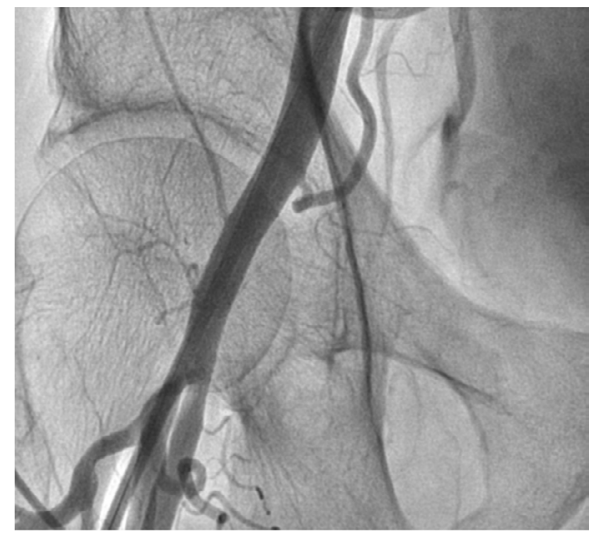

Figure 3: Control angiography of the right external iliac and common femora arteries after sheath exchange and before deployment of the second intraaortic balloon pump, showing no significant lesion.

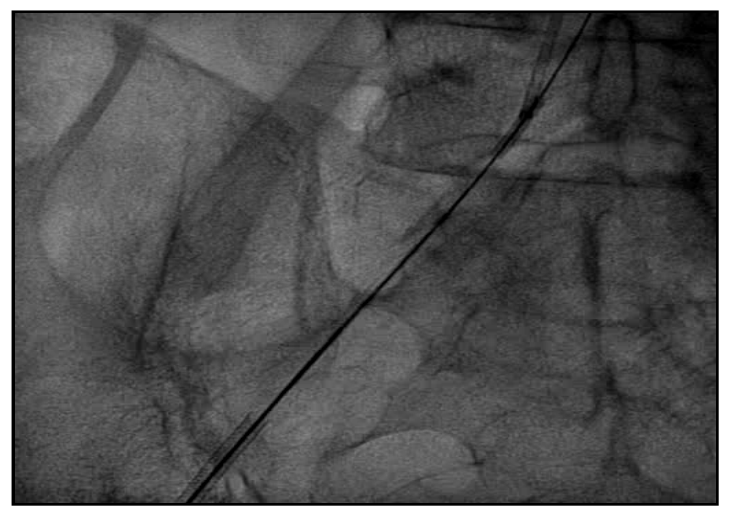

Movie 1: Angiography of the right external iliac and common femoral arteries showing the intra-aortic balloon pump stuck in the delivery sheath.

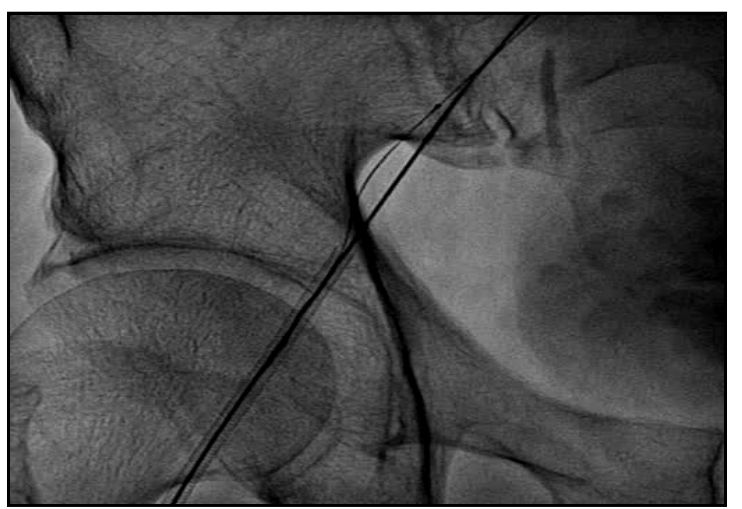

Movie 2: Fluoroscopy showing the 0.018" guide wire (arrow) delivered transradially between the internal wall of the sheath and the intra-aortic balloon pump, toward the right femoral arteriotomy.

with or at risk of cardiogenic shock or those undergoing percutaneous or surgical coronary revascularization [1,4-6]. The ongoing development of this device has enabled refinements in its production, miniaturization of several of its components, and improvements in sensing and triggering features, which make its use appealing despite the availability of other mechanical support devices [7]. Indeed, ease of use and safety represent two important strengths of IABP. Nonetheless, complications still occur with this device, and practitioners should be 
Citation: Zoccai GB, Mancone M, Marullo AGM, Peruzzi M, Cavarretta E, et al. (2013) Intra-aortic Balloon Pump Entrapment in a Transfemoral Sheath: Successful Management with Retrograde Transradial Wiring and Externalization. J Cardiovasc Dis Diagn 1: 103. doi:10.4172/23299517.1000103

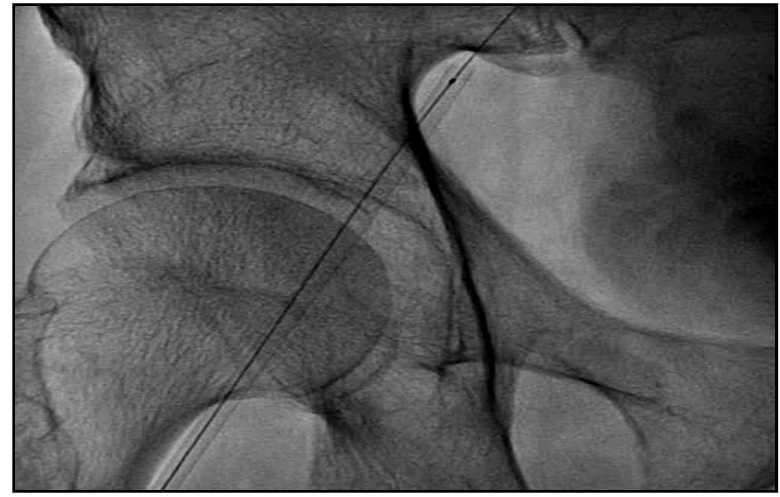

Movie 3: Control angiography of the right external iliac and common femoral arteries after sheath exchange and before deployment of the second intraaortic balloon pump, showing no significant lesion.

aware of their likelihood and the potential remedies.

Entrapment of the IABP balloon is not a novel finding per se, having been reported in several cases, and thus having a non-negligible incidence [8-11]. For instance, Aydin et al. reported on a case of entrapped IABP which remained stuck in the femoral artery after being peeled off from the shaft [12]. Indeed, IABP entrapment often requires surgical intervention and thus represents a potentially life-threatening complication. Our case provides additional insight of this occurrence, and suggests a novel and minimally invasive way to deal with it. It stems from our institution experience in peripheral artery interventions and transcatheter aortic valve implantations, which occasionally require use of exchange-length guide wires with distal tip externalizations and use of such externalized guide wires to deploy other sheaths and catheters. Use of this technique should be straight forward for anyone having minimal endovascular skills and thus could prove beneficial for safe and effective use of IABP in several patients.

\section{References}

1. Perera D, Stables R, Clayton T, De Silva K, Lumley M, et al. (2013) Clack L,
Thomas M, Redwood S (eds.). On behalf of the BCIS-1 Investigators. LongTerm Mortality Data From the Balloon Pump-Assisted Coronary Intervention Study (BCIS-1): A Randomized, Controlled Trial of Elective Balloon Counterpulsation During High-Risk Percutaneous Coronary Intervention. Circulation 127: 207-212.

2. Stone GW, Ohman EM, Miller MF, Joseph DL, Christenson JT, et al. (2003) Contemporary utilization and outcomes of intra-aortic balloon counterpulsation in acute myocardial infarction: the benchmark registry. J Am Coll Cardiol 41:1940-1945.

3. Sangiorgi G, Lauria G, Airoldi F, Godino C, Politi L, et al. (2012) Retrograde popliteal access as bail-out strategy for challenging occlusions of the superficial femoral artery: a multicenter registry. Catheter Cardiovasc Interv 79: 11881193.

4. Thiele H, Zeymer U, Neumann FJ, Ferenc M, Olbrich HG, et al. (2012) Intraaortic balloon support for myocardial infarction with cardiogenic shock. N Engl J Med 367: 1287-1296.

5. Romagnoli E, De Vita M, Burzotta F, Summaria F, Patrizi R, et al. (2012) TCT31 Clinical benefit of radial versus femoral approach in percutaneous coronary intervention with intra-aortic balloon pump support. J Am Coll Cardiol 60: 9-10.

6. Briguori C, Airoldi F, Chieffo A, Montorfano M, Carlino M, et al. (2006) Elective versus provisional intraaortic balloon pumping in unprotected left main stenting. Am Heart J 152: 565-572.

7. de Souza CF, de Souza Brito F, De Lima VC, De Camargo Carvalho AC (2010) Percutaneous mechanical assistance for the failing heart. J Interv Cardio 23:195-202.

8. Papayannis AC, Banerjee S, Brilakis ES (2011) Management of an unusual type of intra-aortic balloon pump dysfunction. J Invasive Cardiol 23: 390

9. Warrillow SJ, Fealy NG, Houston SA (2008) Intra-aortic balloon pump microperforation resulting in balloon entrapment. Anaesth Intensive Care 36 916-917.

10. Brodell GK, Tuzcu EM, Weiss SJ, Simpfendorfer C (1989) Intra-aortic balloonpump rupture and entrapment. Cleve Clin J Med 56: 740-742.

11. Luengtaviboon K, Signhathanadgige S, Chartlaorng B, Surapongse K (2002) Intraaortic balloon entrapment--a rare complication of intraaortic balloon pump. J Med Assoc Thai 85: 153-155.

12. Aydin H, Kandemir O, Günaydin S, Zorlutuna Y (2004) A peeled off and entrapped intraaortic balloon catheter in the femoral artery: an unusual complication. Interact Cardiovasc Thorac Surg 3: 314-316. 